\title{
Cortical Radial Glia: Identification in Tissue Culture and Evidence for Their Transformation to Astrocytes
}

\author{
Susan M. Culican, ${ }^{1}$ Nikki L. Baumrind, ${ }^{1}$ Miyuki Yamamoto, ${ }^{2}$ and Alan L. Pearlman' \\ 'Departments of Cell Biology and Neurology, Washington University School of Medicine, St. Louis, Missouri 63110, and \\ 2Department of Biochemistry, E. K. Shriver Center, Waltham, Massachusetts 02254
}

Radial glia are transiently present in the developing cerebral cortex, where they are thought to guide the migration of neurons from the proliferative zone to the forming cortical plate. To provide a framework for experimental studies of radial glia, we have defined morphological and immunocytochemical criteria to identify them in primary cultures of cortical cells obtained at embryonic day 13 in the mouse. Cortical radial glia in culture for 1-2 d resemble radial glia in vivo: they have a long, thin, unbranched process extending from one or both ends of the elongated cell body and are labeled with the monoclonal antibody RC1 but not with antibodies to glial fibrillary acidic protein (abGFAP). We tested the specificity of $\mathrm{RC} 1$ by double-labeling with a panel of celltype specific antibodies, and found that it labels radial glia, astrocytes, and fibroblast-like cells, but not neurons. Fibroblasts are easily distinguished from glia by morphology and by labeling with antibodies to fibronectin.

To test the hypothesis that radial glia become astrocytes when their developmental role is complete, we examined their morphological and immunocytochemical development in culture. After 3-4 d in vitro radial glia develop several branched processes; in this transitional stage they are labeled by both RC1 and abGFAP. Many radial glia lose RC1 immunoreactivity as they become increasingly branched and immunoreactive to abGFAP. In areas of the cultures that have few neurons and in cultures depleted of neurons by washing, flat, nonprocess-bearing glia predominate. These cells do not lose immunoreactivity to RC1 during the 9-d period of observation even though they acquire GFAP. Thus cortical radial glia undergo an apparently neuron-dependent morphological and immunocytochemical transformation in tissue culture, gradually coming to resemble stellate astrocytes. This in vitro transformation provides support for previous suggestions that a similar event occurs in vivo and opens the prospect for an analysis of the factors that control it.

\footnotetext{
Received July 11, 1989; revised Aug. 30, 1989; accepted Sept. 6, 1989

We are grateful to Jeanette Cohen, William Puckett, and Judy Speck for technical assistance, and to Drs. Virginia Lee, Richard Hynes, John McDonald, and Mark Willard for generously providing antibodies. The study was supported by Research Grant RO1 EY00621 and Training Grant 2-T32 EY07057, both from the National Eye Institute, National Institutes of Health.

Correspondence should be addressed to Dr. Alan L. Pearlman, Department of Cell Biology - Box 8228, Washington University School of Medicine, 660 South Euclid Avenue, St. Louis, MO 63110.
}

Copyright (C) 1990 Society for Neuroscience $0270-6474 / 90 / 100684-09 \$ 02.00 / 0$
Radial glia play an important role in the development of the mammalian CNS and then, with a few prominent exceptions, disappear. Their long, thin processes, which extend radially from the cell body near the ventricle, are thought to guide the migration of the neurons that form the cerebral cortex (Ramón y Cajal, 1911; Rakic, 1972). Radial glia are among the earliest cells to differentiate in the developing cortex (Misson et al., 1988a, b); their transient existence ends shortly after neuronal migration is complete.

What is the fate of this critical cell population? Although cell death is an important mechanism eliminating neurons during cortical development (Finlay and Slattery, 1983; Pearlman, 1985), there is little evidence that radial glia undergo a similar fate. Instead, the presence of transitional cellular forms in Golgistained and immunolabeled cortical sections suggests that radial glia are transformed into astrocytes when they have completed their developmental role (Ramón y Cajal, 1911; Akers, 1977; Schmechel and Rakic, 1979; Levitt et al., 1981; Pixley and de Vellis, 1984; Caviness et al., 1989).

The techniques of cell culture have recently been used effectively in analyzing the fate of early glial populations from several sites in the CNS. In tissue culture, relatively large populations of cells can be sampled at frequent intervals during maturation, and factors that induce or alter cellular maturation can be identified (Raff et al., 1983a, b, 1984, 1988; Hatten, 1985; Fedoroff, 1986; Nagata et al., 1986; McCarthy et al., 1988; Wilkie and Lauder, 1988). Tissue culture studies have also been quite useful in the experimental analysis of the migration of cerebellar neurons along elongated glial processes (Hatten et al., 1984, 1986; Edmondson and Hatten, 1987; Edmondson et al., 1988).

To provide a framework for the study of cortical radial glia in tissue culture, we used immunocytochemical and morphological features to identify radial glia in cultures of cells dissociated from the embryonic cortex of mice. For this identification we used a monoclonal antibody, $\mathrm{RC} 1$, that labels these cells in the developing CNS of the mouse (Edwards et al., 1986, 1990; Misson et al., 1988a). We used a panel of antibodies to test the specificity of $\mathrm{RCl}$ and a polyclonal antibody to glial fibrillary acidic protein (abGFAP) to identify astrocytes. We analyzed the fate of radial glia by studying the changes in their morphology and immunoreactivity that take place over the next several days. In addition, we grew radial glia in cultures depleted of neurons to determine whether the latter play a role in the transformation of radial glia to astrocytes that we obscrved.

\section{Materials and Methods}

Cell culture. The cerebral hemispheres of murine embryos were removed on embryonic day 13 and dissociated (Huettner and Baughman, 1986) 


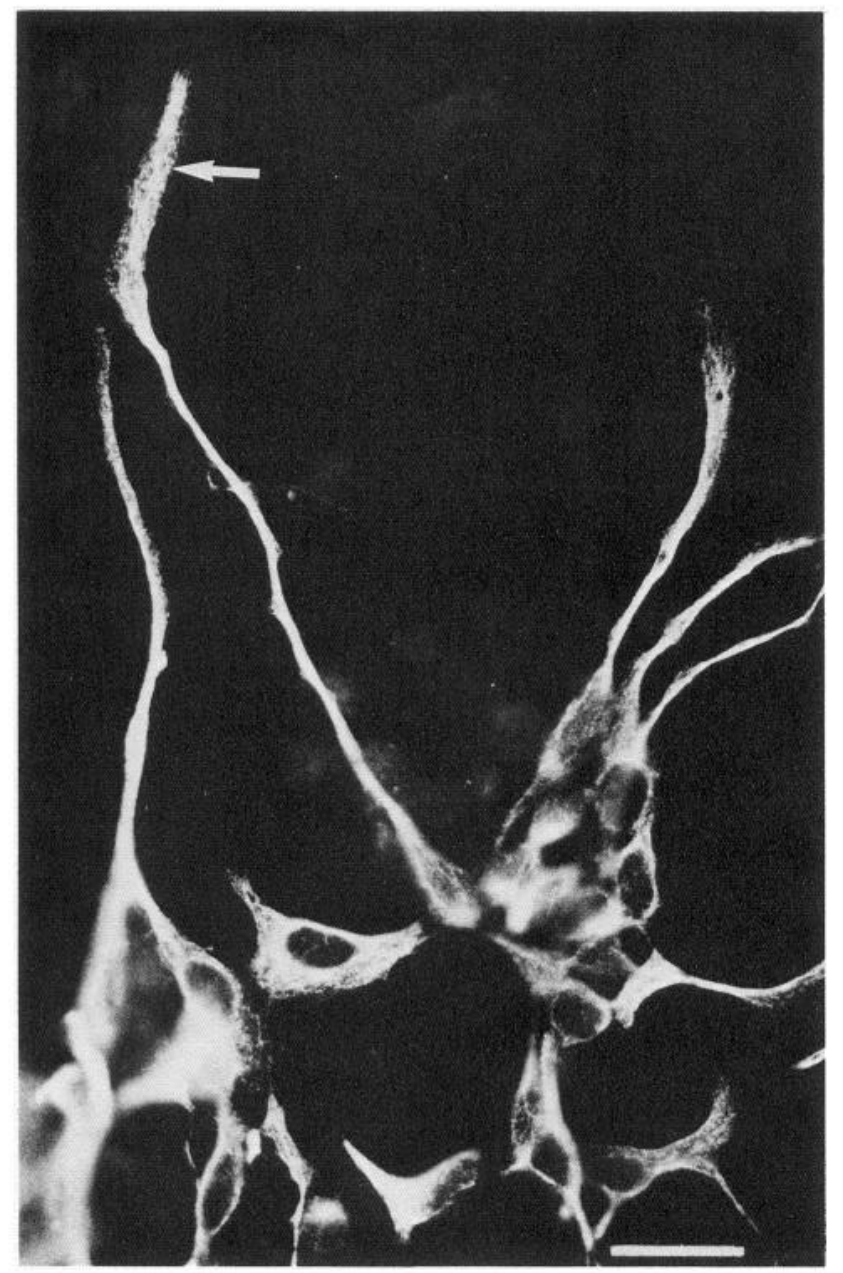

Figure 1. Radial glia at 3 DIV labeled with $\mathrm{RCl}$ and FITC-tagged second antibody. Arrow indicates a broadened, end-foot-like termination of a long, thin process. Scale bar, $20 \mu \mathrm{m}$.

with papain. Dissociated cells were plated (200-300 cells $\left./ \mathrm{mm}^{2}\right)$ on polyL-lysine-coated glass coverslips in 24 well plates and grown in Earle's minimal essential medium (MEM) supplemented with glucose, $10 \%$ heat-inactivated horse serum, and L-glutamine at $35^{\circ} \mathrm{C}$ in $5 \% \mathrm{CO}_{2}$. After 2 days in vitro (DIV), some of the coverslips were washed for $3 \mathrm{sec}$ with cold $\left(4^{\circ} \mathrm{C}\right)$ Earle's MEM to remove neurons, then either fixed immediately or refed and returned to the incubator.

Immunohistochemistry. Cultures were fixed and immunolabeled after $2,4,5,6$, and 9 DIV; 10-36 cultures were double-labeled on each day. Just before fixation, cell cultures were rinsed with L-15 $\left(5 \mathrm{~min}, 35^{\circ} \mathrm{C}\right)$ then fixed in methyl alcohol $\left(5 \mathrm{~min},-70^{\circ} \mathrm{C}\right)$, rinsed, and blocked with fish gelatin ( $1 \%$ in PBS, $30 \mathrm{~min}$; Sigma Chemical). All rinses were carried out with PBS ( $\mathrm{pH} 7.4$ ); primary antibodies were diluted in $0.1 \%$ bovine serum albumin in PBS. Tris-buffered saline was substituted for PBS in rinses and dilutions for immunolabeling with anti-neurofilament antibodies. Immunolabeling was carried out in a dark chamber at room temperature. Two primary antibodies (listed below) were applied simultaneously (90 min); each of the primary antibodies was omitted in labeling controls. Cultures were then rinsed, blocked with fish gelatin, incubated with second antibodies $(60 \mathrm{~min})$, rinsed, and mounted on slides with gelvatol (Rodriquez and Deinhardt, 1960; Monsanto, St. Louis, MO) and 1,4-diazobicyclo(2,2,2)octane (Aldrich Chemicals, Milwaukee, WI) or citifluor (Citifluor Limited, London, England).

The following primary antibodies were used to label cultures: $\mathrm{RC1}$ (undiluted hybridoma supernatant; Edwards et al., 1986, 1989; Mission et al., 1988a), abGFAP (1:4000; Biomed Tech, Stoughton, MA), an affinity-purified polyclonal antibody (rabbit) to human fibronectin (abFN, 1:800, provided by J. McDonald; Villiger et al., 1981), monoclonal and polyclonal antibodies to neurofilaments (abNF; SMI-31, 1:2000, Sternberger-Meyer Immunochemicals, Jarrettsville, MD; RMO-108, 1:10, provided by Dr. V. Lee; Lee et al., 1987; goat anti-rabbit neurofilamentM, 1:100, provided by Dr. M. Willard; Glicksman et al., 1987), and a polyclonal antibody (rabbit) to vimentin (1:1000, provided by R. Hynes; Hynes and Destree, 1978). Second antibodies included rhodamine (TRITC)-conjugated donkey anti-rabbit IgG (H\&L), TRITC-conjugated rabbit anti-mouse IgM, fluorescein(FITC)-conjugated goat antimouse IgG and IgM (Jackson Labs, West Grove, PA), and FITC-conjugated rabbit anti-goat IgG (H\&L) (Cappel Organon Teknika Corp., West Chester, PA). Second antibodies were diluted $1: 200$ in $0.1 \%$ bovine serum albumin in PBS.

A total of 176 cultures were double-labeled for RC1 and GFAP; an additional 25 cultures were double-labeled at 4 DIV to examine the specificity of $\mathrm{RCl}$ immunolabeling. The morphology and immunolabeling of several hundred cells in each culture were examined with an epifluorescence microscope (Nikon Labophot) equipped with a 100-W mercury arc bulb and filter cubes optimized for FITC (Nikon B2) and TRITC (Nikon G1B). Photographs were made with a $35-\mathrm{mm}$ camera (Nikon) on Kodak T-Max film.

\section{Results}

Identification of cortical radial glia in tissue culture: Specificity of immunological markers

An experimental analysis of radial glia in vitro, including a determination of their fate, will require that they be positively identified and distinguished from other cell types present in the cultures. To identify radial glia, we tested 3 antibodies that have been used to label these cells in sections: abGFAP, antibodies to vimentin (abVIM), and the RCl antibody. To distinguish radial glia from other cell types, we used abNF to label neurons and abFN to identify fibroblast-like cells.

In our primary cultures of the dissociated cortical anlage of E13 mice, approximately half of the cells present during the first few DIV have morphological and immunocytological features which suggest that they are radial glia. They have a long, thin, unbranched process extending from one or both ends of the small, elongated cell body (Figs. 1; 2, $a, b ; 4 a$ ). The process sometimes broadens distally to form a structure resembling the radial glial end-foot on the pial basal lamina in vivo. The cytoskeleton of these cells labels strongly with $\mathrm{RCl}$ (Figs. 1, 2b, $4 a$ ), which has been shown to label radial glia in vivo (Edwards et al., 1986, 1990; Misson et al., 1988a). Initially, RC1-positive cells do not label with abGFAP (Fig. 4b), but by 4-6 DIV, most RCl-positive cells are also abGFAP-positive. This transition, which indicates that RC1-positive cells with morphological features of radial glia are related to astroglia, will be considered in more detail in the next section.

We used several monoclonal and polyclonal antibodies (abNF) that label the neurofilament proteins $\mathrm{M}$ and $\mathrm{H}$ to identify neurons. Cells that label with abNF (Fig. 2, $c, d$ ) are not RC1positive, indicating that neurons do not contain the epitope recognized by $\mathrm{RC} 1$. The cells we have identified as radial glia are also abVIM-positive (Fig. 2, $a, b$ ), but this antibody does not reliably distinguish them from neurons, since some of the latter are also abVIM-positive (Fig. 2, $e-h$ ). Fibroblast-like cells, which are also RC1-positive (Fig. $3 a$ ) and abVIM-positive, are large and polygonal; although they are easily distinguished from radial glia morphologically, they sometimes resemble nonprocess-bearing epithelioid glia. We have used 2 established immunocytochemical markers to distinguish these cell types: abGFAP-negative fibroblast-like cells have distinct linear arrays of fibronectin on their surfaces (Fig. 3, $a, b$ ), while abGFAPpositive epithelioid glia do not (Fig. 3, $c, d$ ). 

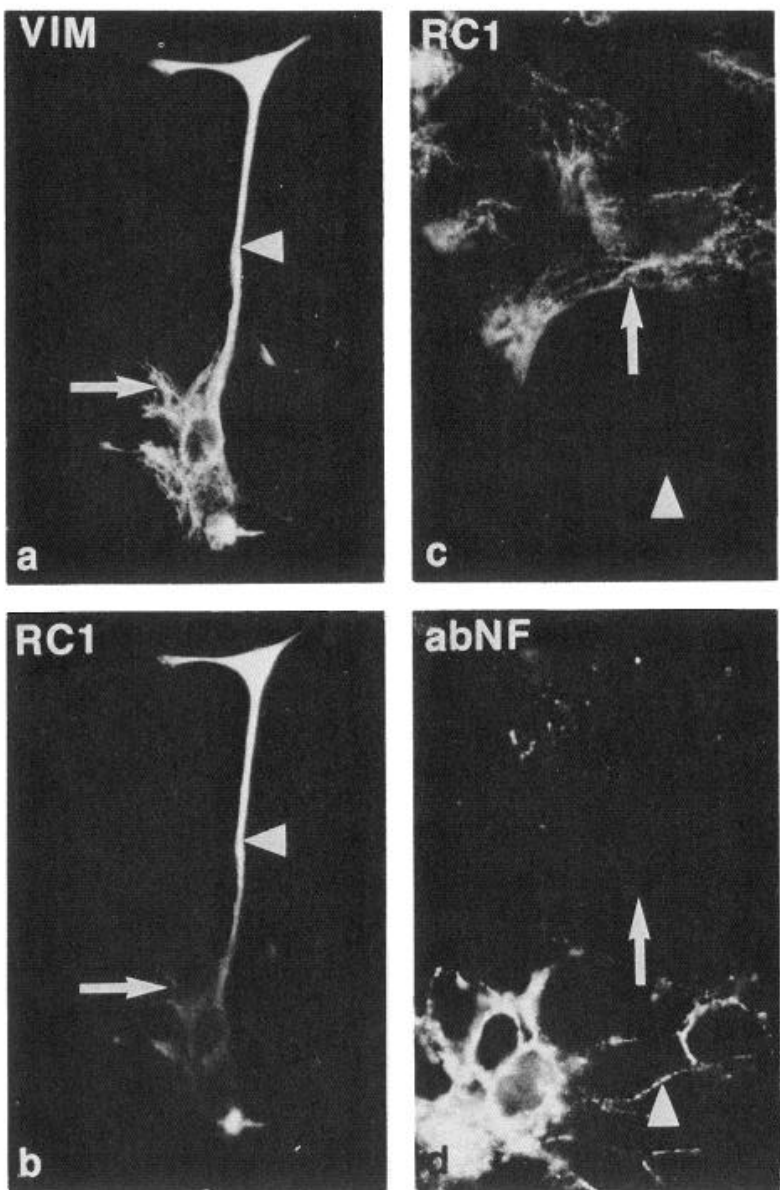
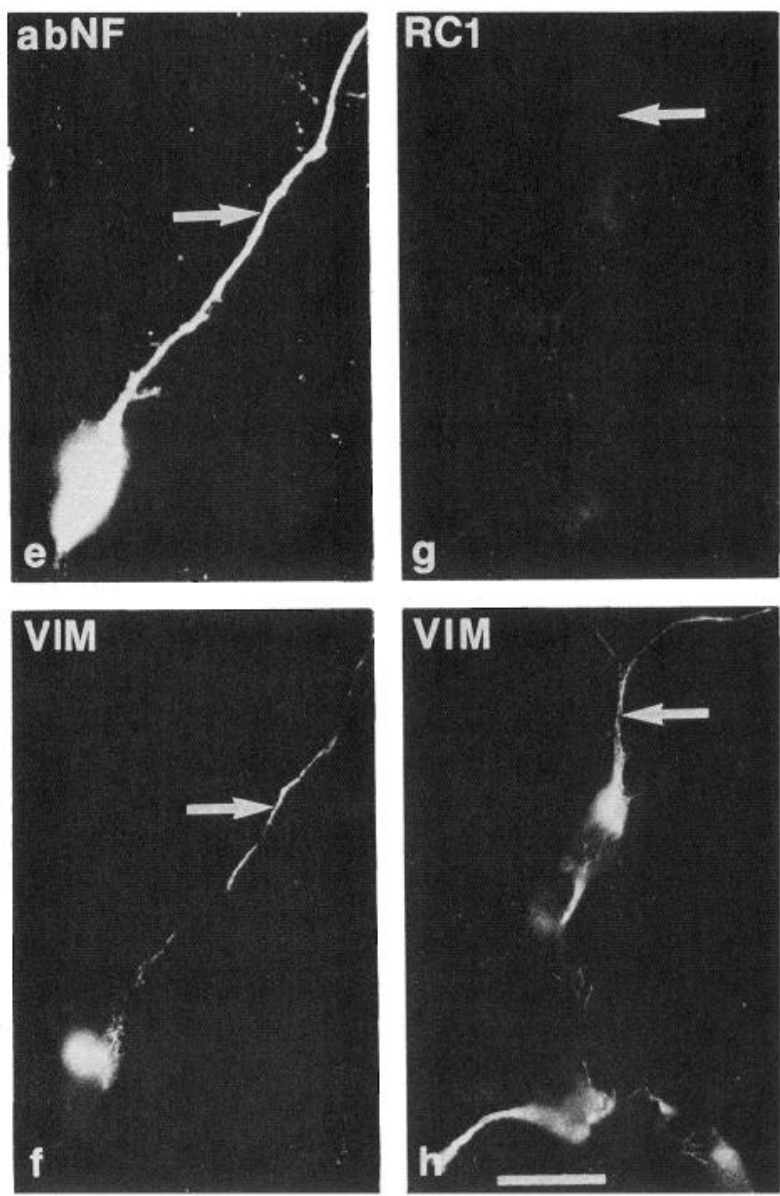

Figure 2. Specificity of immunological markers. Pairs of photographs ( $a$ and $b, c$ and $d$, etc.) are of the same field labeled with the indicated primary antibody and appropriate second antibodies tagged with either FITC or TRITC. Arrows indicate the same point in the 2 photographs. $a$, $b$, A radial glial cell that has a major, elongated, RCl-positive and abVIM-positive process (arrowhead, $a, b$ ) with an end-foot-like termination, and smaller processes emanating from the cell body that are only faintly RC1-positive (arrow, $b$ ) but are strongly VIM-positive (arrow, a). $c, d$, $\mathrm{RC1}$-positive glial cell (arrow, $c$ ) is abNF-negative (arrow, $d$ ), while neuronal processes (arrowhead, $d$ ) are abNF-positive and RCl-negative (arrowhead, $c$ ). $e, f$, A bipolar, abNF-positive neuron (arrow, $e$ ) that is also partially abVIM-positive (arrow, $f$ ). $g, h$, Three cells with neuronal morphology are abVIM-positive (arrow, $h$ ) but RC1-negative (arrow, $g$ ). Scale bar, $20 \mu \mathrm{m}$.

\section{Transformation of radial glia to astrocytes}

To study the morphological and immunocytological changes that take place in radial glia in culture, we double-labeled cultures at 2, 4, 5, 6, and 9 DIV with RC1 and abGFAP. At 2 DIV, approximately half of the cells are RC1-positive; none are abGFAP-positive (Fig. 4, $a, b$ ). Most RC1-positive cells are present, along with $\mathrm{RC} 1$-negative neurons, in small clusters that are the result of both reaggregation and cell division (Luskin et al., 1988) in culture. At this stage, most RC1-positive cells have the appearance of monopolar or bipolar radial glia (Fig. $4 a$ ). Occasional RCl-positive glia have a stellate morphology, with 3 or more relatively short processes that are usually unbranched.

As the cultures mature (4-5 DIV), 2 changes in RC1-positive cells within clusters occur: The proportion of mono- or bipolar radial glia declines while the number of stellate cells increases, and cells of both morphologies display immunoreactivity to abGFAP as well as RC1 (Fig. 4, $c, d$ ). No abGFAP-positive cells are RC1-negative at this stage. With additional time in culture (6-9 DIV) the stellate cells develop more processes and become increasingly immunoreactive to abGFAP. As immunoreactivity to abGFAP increases, labeling with $\mathrm{RC} 1$ decreases; many highly branched stellate astrocytes with prominent abGFAP labeling are RC1-negative (Fig. 4, $e, f$ ).

\section{Neuron-depleted cultures}

In contrast to the RC1-positive radial and stellate glia that are found in clusters with neurons (Fig. 5, $a, b$ ), RC1-positive cells with a flattened, epithelioid morphology and no processes are present in areas of the culture that by chance contain few or no neurons (Fig. 5, $c, d$ ). Some RC1-positive cells, located at the edge of a neuron-glia aggregate or in association with only a few neurons, develop an intermediate morphology: they have welldefined processes, but the processes are broader and the cell body larger and more flattened than stellate cells. Epithelioid glial cells become abGFAP-positive, but, unlike many stellate astrocytes, they retain immunoreactivity to $\mathrm{RC} 1$ throughout the $9 \mathrm{~d}$ of observation.

To test the hypothesis that interaction with neurons is playing a role in determining the morphology of glia, we subjected cultures to a brief, forceful rinse with chilled Earle's MEM at 2 DIV, thereby detaching most neurons. The remaining cells were fixed and labeled with RC1 and abGFAP just after rinsing and at $4,5,6$, and 9 DIV. Immediately after rinsing, the cultures 

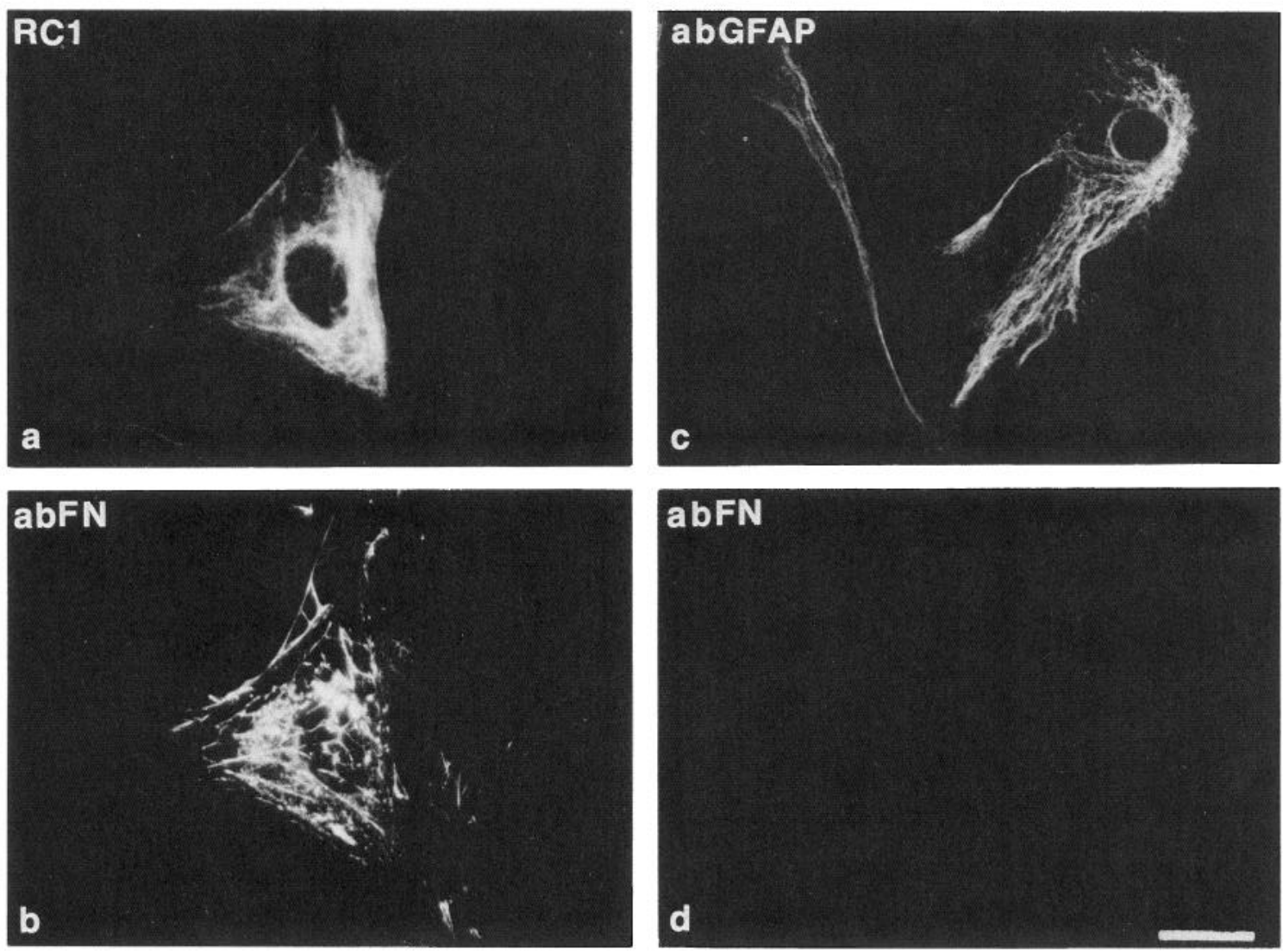

Figure 3. Distinction between fibroblasts and epithelioid glia. Pairs of photographs ( $a$ and $b, c$ and $d)$ are of the same field, double-labeled with the indicated primary antibody, and either FITC- or TRITC-tagged second antibodies. $a, b, \mathrm{RC} 1$-positive flat polygonal fibroblast with fibronectin in linear arrays on its surface. $c, d$, GFAP-positive epithelioid astrocyte with no fibronectin on its surface. Scale bar, $20 \mu \mathrm{m}$.

contain almost exclusively RC1-positive cells that have the morphology of either radial glia (Fig. 6, $a, b$ ) or epithelioid glia. With time in culture (4-9 DIV), radial glia are no longer evident, while epithelioid astrocytes eventually blanket the coverslip. Epithelioid astrocytes develop immunoreactivity to abGFAP but most do not lose immunoreactivity to RC1 (Fig. 6, $c-f$ ).

\section{Discussion}

In this study we provide criteria for identifying radial glia in mixed cultures derived from the embryonic cerebral cortex of the mouse and for differentiating them from other cell types present in the cultures. These criteria are based on the morphology of the cells and their expression of selected antigens; they will undoubtedly require alteration and refinement as new cellular antigens are defined. Radial glia are abundantly present in cultures derived from the early embryonic anlage of the cerebral cortex. Like radial glia in vivo, they have a long, unbranched process extending from one or both ends of the cell body and are labeled by the RCl antibody (Edwards et al., 1986, 1990; Misson et al., 1988a). They are not labeled by abGFAP initially, but become abGFAP-positive after 3-5 d. With time in culture, cells defined as radial glia by these criteria take on the morphological and immunocytological features of stellate astrocytes; they develop several branched processes, become more intensely immunoreactive to abGFAP, and lose immmunoreactivity to $\mathrm{RC} 1$.

\section{$R C 1$ as a label for radial glia}

$\mathrm{RCl}$ is a selective marker for identifying radial glia and astrocytes in tissue culture since it distinguishes these cells from neurons identified with antibodies to neurofilaments. Thus, the apparent specificity of $\mathrm{RCl}$ for radial glia and young astrocytes in tissue sections, where it labels radial glia but not young neurons (Edwards et al., 1986, 1990; Misson et al., 1988a), is preserved in tissue culture. RC1 also labels cells of the meninges in sections (Edwards et al., 1986, 1990; Misson et al., 1988a). The fibroblast-like cells present in our cultures, which are also $\mathrm{RC} 1$-positive and presumably derive from remnants of the meninges, are easily distinguished from radial glia in tissue culture on the basis of morphology and immunoreactivity to anti-fibronectin antibodies. Fibroblasts produce fibronectin and assemble it on their surface in distinct linear arrays (Price and Hynes, 1985). Although fibronectin is produced by both radial glia (Pearlman et al., 1988) and epithelioid astrocytes in culture (Kavinsky and Garber, 1979; Price and Hynes, 1985; Liesi et al., 1986), these cells do not assemble it on their surface in linear arrays (Price and Hynes, 1985; Pearlman et al., 1988) and are thus abFN-negative.

Although RC1 does not label neurons that are expressing the $\mathrm{M}$ or $\mathrm{H}$ components of the neurofilament triplet protein, the question arises as to whether the $\mathrm{RCl}$ epitope might be present in young neurons before they express these markers. Our ob- 

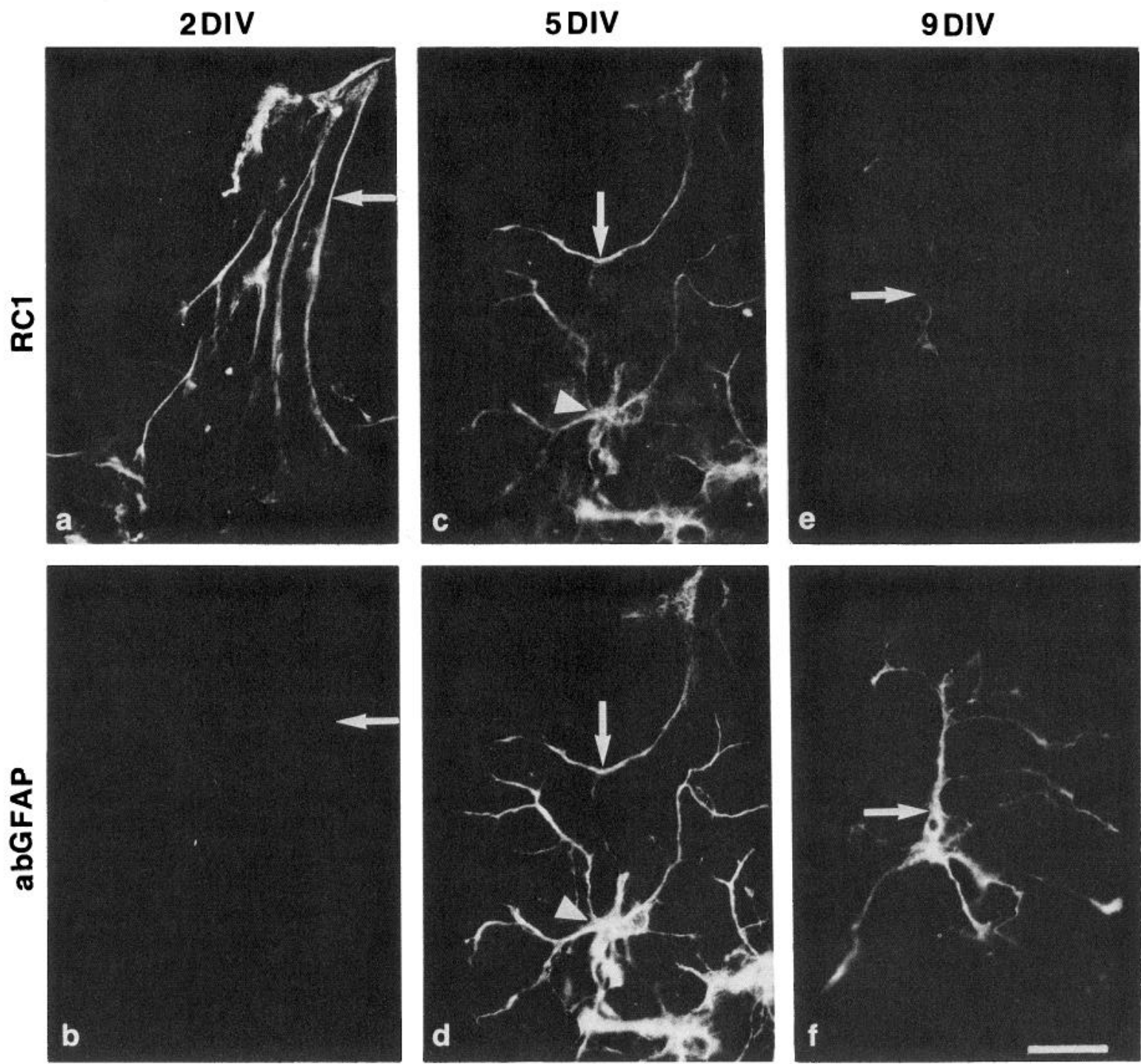

Figure 4. Morphological and immunocytochemical transformation of radial glia. Pairs of photographs $(a$ and $b, c$ and $d, e$ and $f)$ are of the same field double-labeled at the indicated time in culture with RC1 (FITC-tagged second antibody) and abGFAP (TRITC-tagged second antibody). Arrows indicate the same point in the 2 photographs. $a, b$, At 2 DIV, radial glia in cell clusters are mono- or bipolar with elongated, RC1-positive, abGFAP-negative processes (arrow). A few RC1-positive cells have branched processes. $c, d$, By 5 DIV most cells in clusters are stellate, with 3 or more processes, but some (arrow) remain monopolar; most express both the RC1 antigen and GFAP. $e$, $f$, At 9 DIV, most stellate astrocytes have multiple processes and have lost $\mathrm{RCl}$ immunoreactivity. Scale bar, $20 \mu \mathrm{m}$.

servations on the time course of GFAP expression in RC1positive cells suggests that this is not the case. All cells that express GFAP at 3-5 d in culture also express $\mathrm{RC} 1$ to a greater or lesser extent, indicating that the $\mathrm{RC} 1$ epitope may be a component of the cytoskeletal predecessor of GFAP. In contrast, the $\mathrm{RC} 1$ epitope is not detectable at all in abNF-postive cells; if the $\mathrm{RCl}$ epitope were present in early neuronal cells, the transition to more mature neurofilaments would have to be extremely abrupt, which seems unlikely. The appearance of another neuronal marker, neuron-specific enolase, in very young RC1-negative cells and not in RC1-positive cells (S. Culican, J. Sanes, and A. Pearlman, unpublished observations) supports the conclusion that $\mathrm{RC} 1$ does not label neurons.

Antibodies to GFAP, the astrocyte-specific intermediate filament protein, do not label cortical radial glia in tissue culture when they are first plated at E13, just as they do not label radial glia effectively in sections from early embryonic rodents (Big- nami and Dahl, 1974; Bovolenta et al., 1984; Pixley and de Vellis, 1984; Choi, 1988). The late appearance of GFAP in radial glia confirms their relationship to astroglia, but precludes the use of abGFAP as a marker for functional studies of cortical radial glia during the first few days in culture. The usefulness of antibodies to vimentin in such studies is also limited, but for reasons of specificity rather than delayed appearance: we confirm the observation that vimentin is present in both glia and neurons in culture (Bignami et al., 1982). The early appearance of $\mathrm{RC} 1$ and its absence from neurons should make it a suitable marker for functional studies of cortical radial glia similar to those that have been carried out on cerebellar Bergmann glia with abGFAP as the marker (Hatten et al., 1984, 1986; Edmondson and Hatten, 1987; Edmondson et al., 1988).

Vimentin, which was first identified in mesenchymal cells (Lazarides, 1980), is thought to be the major intermediate filament protein in immature neurons and glia as well (Dahl et 

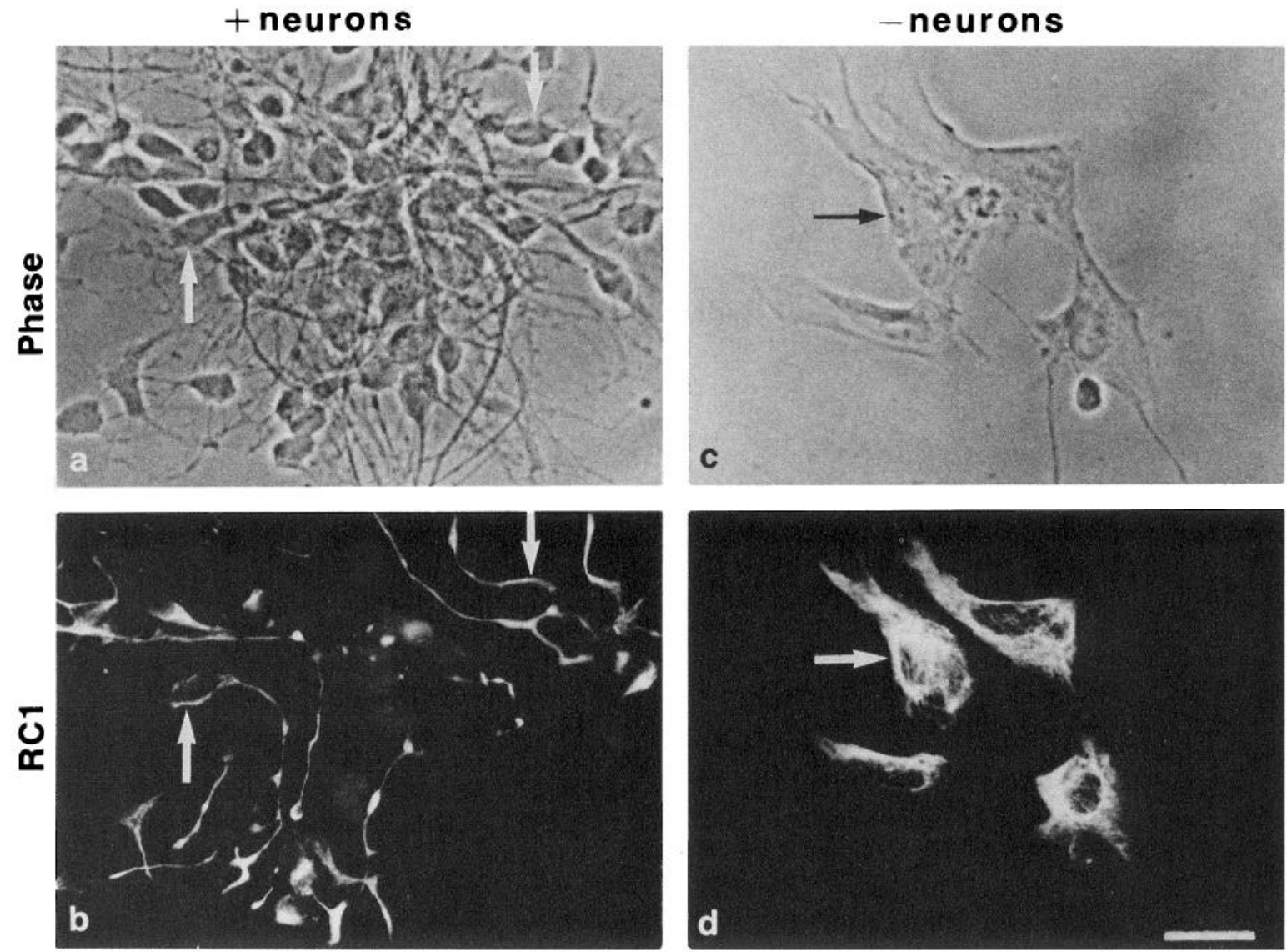

Figure 5. Morphology of glia in clusters with and without adjacent neurons. $a, b$, In clusters with neurons, glial cells have long, thin, RC1-positive processes. $c, d$, In an area of the same culture containing very few neurons, glial cells are epithelioid. Arrows indicate corresponding points in the pairs $(a, b$ and $c, d)$ of phase and immunolabeled images. Scale bar, $20 \mu \mathrm{m}$.

al., 1981; Bignami et al., 1982). It is replaced in most instances by the neurofilament triplet proteins in neurons or by GFAP in astrocytes as these cells mature (Bignami et al., 1982; Bovolenta et al., 1987; for exceptions, see Drager, 1983; Schwob et al., 1986). Vimentin is present in cells of the neuroepithelium at very early stages, before morphological differentiation of neurons and glia takes place (Tapscott et al., 1981; Houle and Fedoroff, 1983). It thus may be the intermediate filament protein of the putative common precursor for neurons and glia. The epitope recognized by the monoclonal antibody RAT 401 is also apparently present in the common precursor and in radial glia (Hockfeld and McKay, 1985). Although there is no evidence for the continued presence of a neuron-glia precursor at E13 (Luskin et al., 1988) when our cultures were obtained, the epitope identified by $\mathrm{RCl}$ is present as early as $\mathrm{E} 10$ in some of the dividing cells in the cortex of the mouse (Misson et al., 1988b). Our evidence indicates that it is limited to the glial cell line once the 2 lines have diverged, but it remains to be determined whether it is also a component of neuroblasts or neuron-glia precursors.

The molecule containing the $\mathrm{RC} 1$ epitope has not yet been identified. The observation that RC1 and abVIM label the cytoskeleton of immature glia and fibroblast-like cells in a similar pattern suggests that $\mathrm{RCl}$ may be identifying a vimentin-like antigen. However, immunoreactivity to the polyclonal abVIM and to the monoclonal antibody $\mathrm{RCl}$ is not completely coextensive in many RC1-positive radial glia (Fig. 2, $a, b$ ), and RC1 immunoreactivity is absent from abVIM-positive cells with neuronal morphology (Fig. 2, $g, h$ ). These findings suggest that $\mathrm{RC} 1$ might be recognizing a molecule (such as an intermediate filament-associated protein; Wang, 1985) that codistributes with vimentin in some cell types but not others.

\section{The radial glia-astrocyte transition in vivo}

Our observations on the transition from radial glia to stellate astrocytes that takes place in tissue culture support the hypothesis that radial glia become astrocytes during cortical development (Ramón y Cajal, 1911; Akers, 1977; Schmechel and Rakic, 1979; Levitt et al., 1981; Pixley and de Vellis, 1984; Caviness et al., 1989). This hypothesis stems from morphological observations during the period when radial glia disappear from the cortex and astrocytes become prominent. During this period, cell forms are evident that appear to be transitional because they have a long radial process extending to the pia but also have an astrocyte-like cell body outside of the ventricular and subventricular zones with several locally branching processes (Ramón y Cajal, 1911; Akers, 1977; Schmechel and Rakic, 1979; Levitt et al., 1981; Choi, 1988; Misson et al., 1988b; Caviness et al., 1989). A similar set of observations has led to the proposal that radial glia are transformed into both astrocytes and Bergmann glia in the cerebellum (Ramón y Cajal, 1911; Rakic, 1971; Choi and Lapham, 1978, 1980; Bovolenta et al., 1984; BenjellounTouimi et al., 1985). However, neither the studies of radial glia in sections nor our studies in tissue culture provide evidence as 

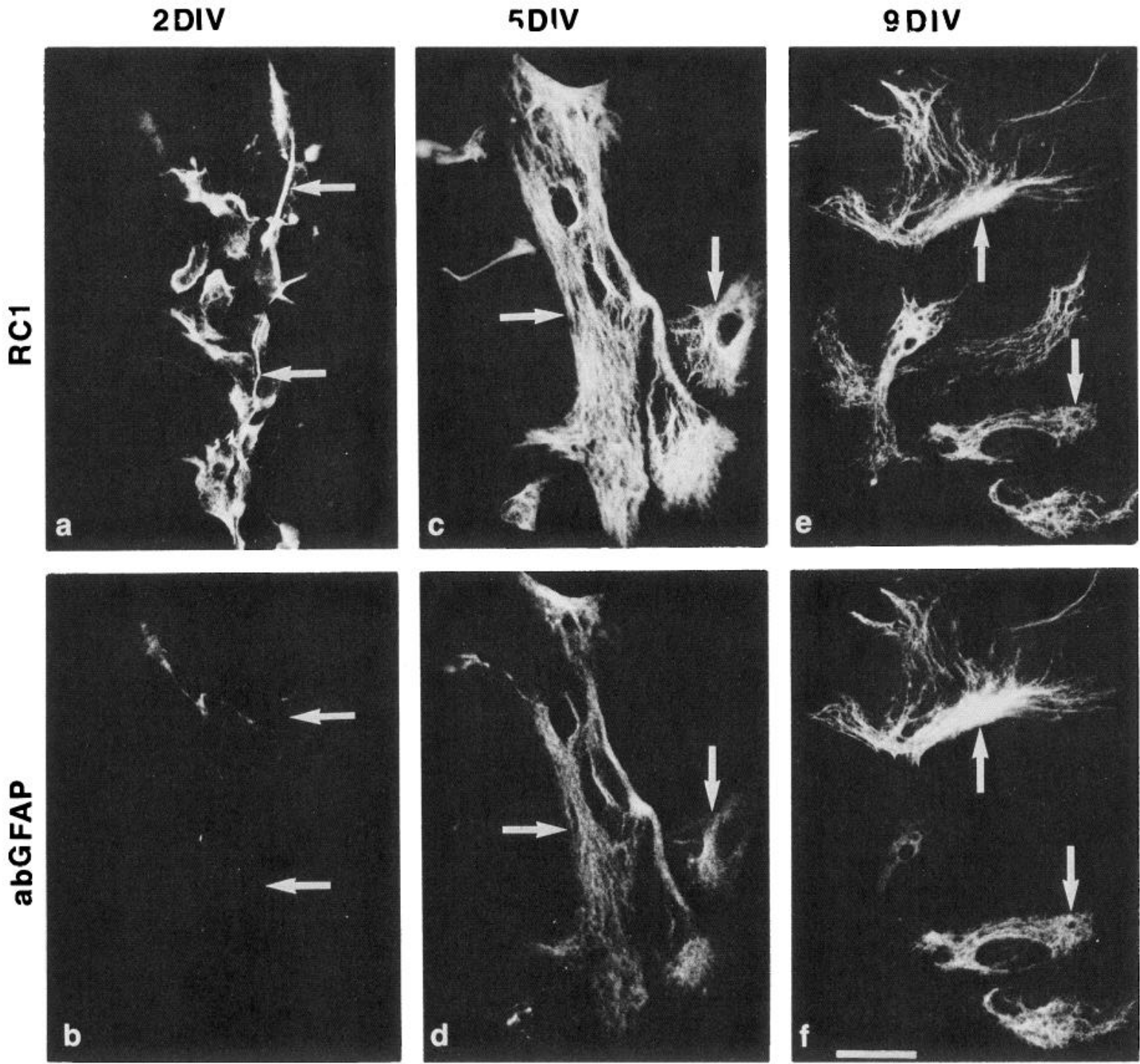

Figure 6. Neuron-depleted cultures. Pairs of photographs ( $a$ and $b, c$ and $d, e$ and $f$ ) are of the same field double-labeled as in Figure 2 with RC1 and abGFAP after the indicated time in culture. $a, b$, Immediately after a brief rinse with chilled Earle's MEM, some RC1-positive, abGFAPnegative radial glia-like cells remain (arrows), their morphology altered by the treatment. $c, d$, At 5 DIV, the predominant cell type is a flattened, epithelioid astrocyte with prominent labeling of the cytoskeleton with RCl and definite but less prominent labeling with abGFAP (arrows). $e, f$, By 9 DIV most epithelioid astrocytes are abGFAP-positive, but have not lost RC1 immunoreactivity (arrows). Scale bar, $20 \mu \mathrm{m}$.

to what proportion of the radial glia become astrocytes, or what proportion of the astrocytes are derived from radial glia.

Several studies have suggested that radial glia might also be transformed into oligodendroglia in the spinal cord, based on the observation of cells that are transitional in morphology and immunolabeling (Penfield, 1924; Ramon-Moliner, 1958; Choi et al., 1983; Hirano and Goldman, 1988). The mechanism for oligodendroglial production may differ in the telencephalon, however, where the cells of the subventricular zone apparently give rise to oligodendroglia independent of the radial glia-astrocyte line (LeVine and Goldman, 1988).

\section{In vitro analysis of glial differentiation and fate}

In zones of the cultures that have few neurons and in cultures that have been depleted of neurons by washing, flat, epithelioid astrocytes with no processes are present rather than astrocytes with several processes. Although epithelioid astrocytes are labeled by abGFAP, they do not lose immunoreactivity to $\mathrm{RC} 1$ during the period we have studied (E13; 2-9 DIV), suggesting that both the development of glial processes and the development of the mature cytoskeletal composition are dependent on interactions with neurons.

Our observations are in agreement with prior studies demonstrating that contact with neurons plays an important role in determining astroglial morphology in vitro. Early postnatal cerebellar astrocytes grown in the absence of neurons assume a flat, epithelioid morphology, but can be transformed into stellate astrocytes or cells resembling Bergmann glia by the addition of purified granule cells (Hatten, 1985, 1987; Nagata et al., 1986). In contrast, cultures of dissociated cells from the midbrain that contain relatively few neurons have many stellate astrocytes, some of which are transformed radial glia (Wilkie and Lauder, 1988). Astrocytic proliferation is strikingly arrested by direct contact with neurons or neuronal membranes (Sobue and Pleasure, 1984; Hatten, 1987), but not by media conditioned by neurons, glia, or both (Hatten, 1985). 
Tissue culture has proven to be an effective means for analyzing the development of glial cells (Trimmer et al., 1982; Mason et al., 1988), transformations in their morphology (reviewed in Fedoroff, 1986, and Lauder and McCarthy, 1986), and factors that influence the fate of glial precursors (Raff et al., 1983a, b, 1984, 1988; Hughes et al., 1988). Our observation that the transformation of cortical radial glia to astrocytes occurs in vitro opens the prospect for an analysis of the mechanisms that control this process during early cortical development.

\section{References}

Akers, R. M. (1977) Radial fibers and astrocyte development in the rat cerebral cortex. Anat. Rec. 187: 520-521.

Benjelloun-Touimi, S., C. M. Jacque, P. Derer, F. DeVitry, R. Maunoury, and P. Dupoucy (1985) Evidence that mouse astrocytes may be derived from the radial glia. An immunohistochemical study of the cerebellum in the normal and reeler mouse. J. Neuroimmunol. 9:8797.

Bignami, A., and D. Dahl (1974) Astrocyte-specific protein and radial glia in the cerebral cortex of newborn rat. Nature 252: 55-56.

Bignami, A., T. Raju, and D. Dahl (1982) Localization of vimentin, the nonspecific intermediate filament protein, in embryonal glia and in early differentiating ncurons. Dcv. Biol. 91: 286-295.

Bovolenta, P., R. K. H. Liem, and C. A. Mason (1984) Development of cerebellar astroglia: Transitions in form and cytoskeletal content. Dev. Biol. 102: 248-259.

Bovolenta, P., R. K. H. Liem, and C. A. Mason (1987) Glial filament protein expression in astroglia in the mouse visual pathway. Dev. Brain Res. 33: 113-126.

Caviness, V. S., Jr., J.-P. Misson, T. Takashi, and J. E. Crandall (1989) Astroglial transformations in the developing murine cerebral wall. Soc. Neurosci. Abstr. 15: 328.

Choi, B. H. (1988) Prenatal gliogenesis in the developing cerebrum of the mouse Glia 1: 308-316.

Choi, B. H., and L. W. Lapham (1978) Radial glia in the human fetal cerebellum: A combined golgi, immunofluorescent and electron microscopic study. Brain Res. 148: 295-311.

Choi, B. H., and L. W. Lapham (1980) Evolution of Bergmann glia in developing human fetal cerebellum: A golgi, electron microscope and immunofluorescent study. Brain Res. 190: 369-383.

Choi, B. H., R. C. Kim, and L. W. Lapham (1983) Do radial glia give rise to both astroglial and oligodendroglial cells? Dev. Brain Res 8 : 119-130.

Dahl, D., D. C. Rueger, and A. Bignami (1981) Vimentin, the 57,000 molecular weight protein of fibroblast filaments, is the major cytoskeletal component in immature glia. Eur. J. Cell Biol. 24: 191-196.

Drager, U. C. (1983) Coexistence of neurofilaments and vimentin in a neurone of adult mouse retina. Nature 303: 169-172.

Edmondson, J. C., and M. E. Hatten (1987) Glial-guided granule neuron migration in vitro: A high-resolution time-lapse video microscopic study. J. Neurosci. 7: 1928-1934.

Edmondson, J. C., R. K. H. Liem, J. E. Kuster, and M. E. Hatten (1988) Astrotactin: A novel neuronal cell surface antigen that mediates neuron-astroglial interactions in cerebellar microcultures. J. Cell Biol. 106: 505-517.

Edwards, M. A., M. Yamamoto, G. Schwarting, and V. S. Caviness, Jr. (1986) Development of radial glia in the mouse; an immunohistochemical study with a cell-class specific monoclonal antibody. Soc. Neurosci. Abstr. 12: 182

Edwards, M. A., M. Yamamoto, and V. S. Caviness, Jr. (1990) Organization of radial glia and related cells in the developing murine CNS. An analysis based upon a new monoclonal antibody marker. Neuroscience (in press).

Fedoroff, S. (1986) Astrocytes: Development, Morphology, and Regional Specialization of Astrocytes, pp. 35-74, Academic, Orlando, FL.

Finlay, B., and M. Slattery (1983) Local differences in the amount of early cell death in neocortex predict adult local specializations. Science 219: 1349-1351.

Glicksman, M. A., D. Soppet, and M. B. Willard (1987) Posttranslational modification of neurofilament polypeptides in rabbit retina. J. Neurobiol. 18: 167-196.
Hatten, M. E. (1985) Neuronal regulation of astroglial morphology and proliferation in vitro. J. Cell Biol. 100: 384-396.

Hatten, M. E. (1987) Neuronal inhibition of astroglial cell proliferation is membrane mediated. J. Cell Biol. 104: 1353-1360.

Hatten, M. E., R. K. Liem, and C. A. Mason (1984) Defects in specific associations between astroglia and neurons occur in microcultures of weaver mouse cerebellar cells. J. Neurosci. 4: 1163-1172.

Hatten, M. E., R. K. H. Liem, and C. A. Mason (1986) Weaver mouse cerebellar granule neurons fail to migrate on wild-type astroglial processes in vitro. J. Neurosci. 6: 2676-2683.

Hirano, M., and J. E. Goldman (1988) Gliogenesis in rat spinal cord: Evidence for origin of astrocytes and oligodendrocytes from radial precursors. J. Neurosci. Res. 21: 155-167.

Hockfield, S., and R. D. G. McKay (1985) Identification of major cell classes in the developing mammalian nervous system. J. Neurosci. 5: 3310-3328.

Houle, J., and S. Fedoroff (1983) Temporal relationship between the appearance of vimentin and neural tube development. Dev. Brain Res. 9: 189-195.

Huettner, J. E., and R. W. Baughman (1986) Primary culture of identified neurons from the visual cortex of postnatal rats. J. Neurosci. 6: 3044-3060.

Hughes, S. M., L. E. Lillien, M. C. Raff, H. Rohrer, and M. Sendtner (1988) Ciliary neurotrophic factor induces type-2 astrocyte differentiation in culture. Nature 335: 70-73.

Hynes, R. O., and A. T. Destree (1978) $10 \mathrm{~nm}$ filaments in normal and transformed cells. Cell 13: 151-163.

Kavinsky, C. J., and B. B. Garber (1979) Fibronectin associated with the glial component of embryonic brain cell cultures. J. Supramol. Struct. 11: 269-281.

Lauder, J., and K. McCarthy (1986) Neuronal-glial interactions. In Astrocytes. Vol. 2. Biochemistry, Physiology, and Pharmacology of Astrocytes, S. Fedoroff and A. Vernadakis, eds., pp. 295-314, Academic, Orlando, FL.

Lazarides, E. (1980) Intermediate filaments as mechanical integrators of cellular space. Nature 283: 249-256.

Lee, V. M. Y., M. J. Carden, W. W. Schlappfer, and J. Q. Trojanowski (1987) Monoclonal antibodies distinguish several differentially phosphorylated states of the two largest rat neurofilament subunits (NF-H and NF-M) and demonstrate their existence in the normal nervous system of adult rats. J. Neurosci. 7: 3474-3488.

LeVine, S. M., and J. E. Goldman (1988) Embryonic divergence of oligodendrocyte and astrocyte lineages in developing rat cerebrum. J. Neurosci. 8: 3992-4006.

Levitt, P., M. L. Cooper, and P. Rakic (1981) Coexistence of neuronal and glial precursor cells in the cerebral ventricular zone of the fetal monkey: An ultrastructural immunoperoxidase analysis. J. Neurosci. 1: $27-39$.

Liesi, P., T. Kirkwood, and A. Vaheri (1986) Fibronectin is expressed by astrocytes cultured from embryonic and early postnatal rat brain. Exp. Cell. Res. 163: 175-185.

Luskin, M. B., A. L. Pearlman, and J. R. Sanes (1988) Cell lineage in the cerebral cortex of the mouse studied in vivo and in vitro with a recombinant retrovirus. Neuron $1: 635-647$.

Mason, C. A., J. C. Edmondson, and M. E. Hatlen (1988) The extending astroglial process: Development of glial cell shape, the growing tip, and interactions with neurons. J. Neurosci. 8: 3124-3134.

McCarthy, K. D., A. Salm, and L. S. Lerea (1988) Astroglial receptors and their regulation of intermediate filament protein phosphorylation. In Glial Cell Receptors, H. K. Kimelberg, ed., pp. 1-34, Raven, New York.

Misson, J.-P., M. A. Edwards, M. Yamamoto, and V. S. Caviness, Jr. (1988a) Identification of radial glial cells within the developing murine central nervous system: Studies based upon a new immunohistochemical marker. Dev. Brain Res. 44: 95-108.

Misson, J.-P., M. A. Edwards, M. Yamamoto, and V. S. Caviness, Jr. (1988b) Mitotic cycling of radial glial cells of the fetal murine cerebral wall: A combined autoradiographic and immunohistochemical study. Dev. Brain Res. 38: 183-190.

Nagata, I., G. Keilhauer, and M. Schachner (1986) Neuronal influence on antigenic marker profile, cell shape and proliferation of cultured astrocytes obtained by microdissection of distinct layers from the early postnatal mouse cerebellum. Dev. Brain Res. 24: 217-232.

Pearlman, A. L. (1985) The visual cortex of the normal mouse and the reeler mutant. In Cerebral Cortex. Vol 3: Visual Cortex, A. Peters and E. G. Jones, eds., pp. 1-17, Plenum, New York. 
Pearlman, A. L., J. Cohen, and W. Puckett (1988) Radial glia: A cellular source for fibronectin during early cortical development. Soc. Neurosci. Abstr. 11: 748.

Penfield, W. (1924) Oligodendroglia and its relation to classical neuroglia. Brain 17: 430-452.

Pixley, S. R., and J. de Vellis (1984) Transition between immature radial glia and mature astrocytes studied with a monoclonal antibody to vimentin. Dev. Brain Res. 15: 201-209.

Price, J., and R. O. Hynes (1985) Astrocytes in culture synthesize and secrete a variant form of fibronectin. J. Neurusci. 5: 2205-2211.

Raff, M. C., E. R. Abney, J. Cohen, R. Lindsay, and M. Noble (1983a) Two types of astrocytes in cultures of developing rat white matter: Differences in morphology, surface gangliosides, and growth characteristics. J. Neurosci. 3: 1289-1300.

Raff, M. C., R. H. Miller, and M. Noble (1983b) A glial progenitor cell that develops in vitro into an astrocyte or an oligodendrocyte depending on culture medium. Nature 303: 390-396.

Raff, M. C., E. R. Abney, and R. H. Miller (1984) Two glial cell lineages diverge prenatally in rat optic nerve. Dev. Biol. 106: 53-60.

Raff, M. C., L. E. Lillien, W. D. Richardson, J. F. Burne, and M. D. Noble (1988) Platelet-derived growth factor from astrocytes drives the clock that times oligodendrocyte development in culture. Nature 333: 562-565.

Rakic, P. (1971) Neuron-glia relationship during granule cell migration in developing cerebellar cortex. A Golgi and electromicroscopic study in macacus rhesus. J. Comp. Neurol. 141: 283-312.

Rakic, P. (1972) Mode of cell migration to the superficial layers of fetal monkey neocortex. J. Comp. Neurol. 145: 61-84.

Ramón y Cajal, S. (1911) Histologie du systeme nerveux de l'homme et des vertebres, Maloine, Paris.
Ramon-Moliner, E. (1958) A study on neuroglia. The problem of transitional forms. J. Neurosci. Res. 21: 155-167.

Rodriguez, J., and F. Deinhardt (1960) Preparation of a semipermanent mounting medium for fluorescent antibody studies. Virology 12: 316-317.

Schmechel, D. E., and P. Rakic (1979) A golgi study of radial glial cells in developing monkey telencephalon: Morphogenesis and transformation into astrocytes. Anat. Embryol. 156: 115-152.

Schwob, J. E., N. B. Farber, and D. I. Gottlieb (1986) Neurons of the olfactory epithelium in adult rats contain vimentin. J. Ncurosci. 6 : 208-217.

Sobue, G., and D. Pleasure (1984) Astroglial proliferation and phenotype are modulated by neuronal plasma membrane. Brain Res. 324: $175-179$

Tapscott, S. J., G. S. Bennett, F. Toyama, F. Kleinbart, and H. Holtzer (1981) Intermediate filament proteins in the developing chick spinal cord. Dev. Biol. 86: 40-54.

Trimmer, P. A., P. J. Reier, T. H. Oh, and L. F. Eng (1982) An ultrastructural and immunocytochemical study of astrocyte differentiation in vitro. J. Neuroimmunol. 2: 235-260.

Villiger, B., D. G. Kelley, W. Engleman, C. Kuhn, and J. A. McDonald (1981) Human alveolar macrophage fibronectin: Synthesis, secretion, and ultrastructural localization during gelatin-coated latex particle binding. J. Cell Biol. 90: 711-720.

Wang, E. (1985) Intermediate filament associated proteins. Ann. NY Acad. Sci. 455: 32-56.

Wilkie, M., and J. M. Lauder (1988) A simple method for the culture of glial cells from embryonic rat brain: Implications for regional heterogeneity and the radial glial lineage. J. Neurosci. Res. $21: 220-225$. 